Objective: To determine rates and predictors of RSV testing during bronchiolitis care episodes.

Design/methods: Retrospective cohort study involving 125,031 infants $\geq 32$ weeks gestation born at 6 Kaiser Permanente hospitals 1996-2002. We defined an episode as a time period that:

1) included selected diagnosis codes (e.g., 466.1 acute bronchiolitis),

2) began with a related diagnosis code 2 days prior to a specific bronchiolitis code, and

3) ended with a relevant diagnosis code followed by 14 clear days.

Results: Of 21,789 bronchiolitis episodes of care in our cohort, 4275 (19.6\%) had at least one test for RSV. In multivariate analyses, gestational age 34-36 weeks, hospitalization status, and length of episode between 2-13 days predicted testing status, with adjusted odds ratios of $1.23(95 \% \mathrm{Cl}, 1.07,1.41)$, $17.6(15.3,20.1)$ and $3.49(3.20,3.80)$, respectively. Overall, $45.5 \%$ of RSV tests were positive.

Conclusions: Only a small portion of infants with bronchiolitis are tested for RSV, leading to an underdiagnosis of RSV by approximately $80 \%$. The strongest predictors of testing status are episode length and whether the episode includes a hospitalization.

The study was sponsored by Medlmmune.

180

\section{THE TRANSMISSION RATE OF CYTOMEGALOVIRUS TO EXTREMELY PRETERM INFANTS IS NOT REDUCED BY ROUTINE FREEZING OF MATERNAL MILK}

S. Omarsdottir ${ }^{1}$, C. Printz ${ }^{1}$, F. Gustafsson ${ }^{1}$, L. Legnevall ${ }^{1}$, B. Zweygberg-Wirgart ${ }^{2}$, L. Grillner², H. Lagercrantz ${ }^{3}, M$. Vanpée ${ }^{1}$

${ }^{1}$ Neonatal Unit, Karolinska Institute, Karolinska University Hospital, ${ }^{2}$ Clinical Microbiology, Karolinska University Hospital, ${ }^{3}$ Karolinska Institute, Stockholm, Sweden

Background and aims: Breastmilk induced cytomegalovirus (CMV) transmission can cause symptomatic disease in the extremely preterm infant (EPI). The aim of this study was to evaluate if routine freezing of maternal milk reduces CMV transmission and disease.
Methods: One hundred forty EPIs (< 28 weeks) and their 127 mothers were included. Infants were randomized to be fed only freeze-thawed maternal milk (FTM) or both fresh and freeze-thawed maternal milk (F+FTM). FTM was frozen at $-20^{\circ} \mathrm{C}$ for $\geq 3$ days. In mothers CMV serology was taken and maternal milk was analyzed using CMV-PCR and CMV culture. In EPIs CMV was analyzed in urine by PCR and culture until 6-12 weeks post partum. Signs and laboratory findings were documented in infants infected by CMV.

Results: Sixty-nine EPIs were randomized to be fed FTM whereas 71 were randomized to be fed F+FTM. CMV serology was taken in 116 mothers and in $81(70 \%)$ CMV-serology was positive. Of the CMV seropositive mothers, breastmilk was positive for CMV-DNA in $66(81 \%)$ and CMV culture was positive in $56(70 \%)$. CMV was transmitted to 4 of $37(11 \%)$ EPIs that were fed CMV-positive FTM and to 2 of $34(6 \%)$ EPIs that were fed CMV-positive F+FTM (NS). No infant developed clinical signs of CMV disease. However, transient hepatic affection occured in 2 infants in the FTM group and in 1 infant in the F+FTM group .

Conclusions: Routine freezing of maternal milk did not reduce the risk for CMV transmission or symptomatic disease in the EPI.

\section{1}

\section{INTERFERON-Г, INTERFERON-Г-INDUCIBLE PROTEIN-10 AND TUBERCULIN SKIN TEST RESPONSES OF HIV-POSITIVE AND HIV- NEGATIVE CHILDREN IN NORTHEAST BRAZIL}

N. Cavalcanti ${ }^{1}$, S. Bihari ${ }^{2}$, J. Correia ${ }^{1}$, G. Alves ${ }^{1}$, E. Souza1', B. Brabin², L. Cuevas ${ }^{2,3}$

${ }^{1}$ Instituto de Medicina Integral Professor Fernando Figueira, Recife, Brazil, ${ }^{2}$ Liverpool School of Tropical Medicine, Liverpool, UK, ${ }^{3}$ World Health Organization, Geneva, Switzerland

Background and aims: Detection of latent tuberculosis (TB) infection (LTBI) and provision of chemoprophylaxis is an important strategy for TB control, particularly for HIV-infected children. Assessing LTBI in this population is difficult because the tuberculin skin test (TST) is unreliable in immunosuppressed individuals. Interferon- $\gamma$ release assays (QTF) have been recently used as complementary tools for the diagnosis of LTBI and Interferon- $y$ inducible protein-10 (IP-10) has been evaluated as a potential marker for detection of Mycobacterium tuberculosis infection. Our aim was 